\title{
Avaliação da biocompatibilidade da membrana do dialisador em cães com insuficiência renal aguda induzida por gentamicina tratados por hemodiálise
}

\author{
Evaluation of the biocompatibility of the dialyzer membrane in dogs with acute kidney injury
}

induced by gentamicin treated by hemodialysis

Evaluación de la biocompatibilidad de la membrana del dializador en perros con insuficiencia renal inducida por gentamicina tratados mediante hemodiálises

Andre Marcelo Conceição Meneses

ORCID: https://orcid.org/0000-0003-3494-2998

Universidade Federal Rural da Amazônia, Brasil

Escola Universitária Vasco da Gama, Portugal

E-mail: andre.meneses1974@icloud.com

Elisa Cunha Pereira

ORCID: https://orcid.org/0000-0001-7231-0057

Universidade Federal Rural da Amazônia, Brasil

E-mail: elisa_cunhap@hotmail.com

Alessandra Melchert

ORCID: https://orcid.org/0000-0002-8680-2121

Universidade Estadual Paulista "Júlio de Mesquita Filho", Brasil E-mail: alessandra.melchert@unesp.br

João Roberto de Araujo Caldeira Brant

ORCID: https://orcid.org/0000-0001-5076-5907

Médico Veterinário Autônomo

E-mail: jrbrantvet@gmail.com

Pasqual Barretti

ORCID: https://orcid.org/0000-0003-4979-4836 Universidade Estadual Paulista "Júlio de Mesquita Filho", Brasil

E-mail: pbarretti@uol.com.br

Regina Kiomi Takahira

ORCID: https://orcid.org/0000-0003-3323-4199 Universidade Estadual Paulista "Júlio de Mesquita Filho", Brasil

E-mail: regina.takahira@unesp.br

Ednaldo da Silva Filho

ORCID: https://orcid.org/0000-0002-8009-3504

Universidade Federal Rural da Amazônia, Brasil

E-mail: tuca13@yahoo.com

Jacqueline Costa Teixeira Caramori

ORCID: https://orcid.org/0000-0002-0093-9515 Universidade Estadual Paulista "Júlio de Mesquita Filho", Brasil E-mail: j.camori@unesp.br

\begin{abstract}
Resumo
Procedimentos dialíticos como a hemodiálise (HD) procuram sustentar a vida do paciente com insuficiência real aguda (IRA), até que haja recuperação da função renal. Este trabalho tem como objetivo avaliar a biocompatibilidade das membranas de acetato de celulose e polissulfona em cães sem e com IRA submetidos à hemodiálise, através das respostas biológicas e sinais clínicos presentes no processo. Os animais hemodialisados foram subdivididos em quatro grupos (8 animais em cada): grupo 1 composto por animais normais que usaram membrana de acetato de celulose; grupo 2 formado por animais com IRA que utlizaram acetato de celulose; grupo 3 composto por animais normais que utilizaram membrana de polissulfona; e grupo 4 formado por animais com IRA que usaram polissulfona. A indução da IRA foi realizada pela administração de gentamicina $(45 \mathrm{mg} / \mathrm{kg} / \mathrm{EV} /$ dia). A principal alteração foi perda de peso dos animais dos grupos com IRA. Houve redução significativa nos níveis de ureia e creatinina principalmente nos animais hemodialisados com membrana de polissulfona, enquanto que os níveis de sódio, potássio e glicose não apresentaram diferenças significativas entre os grupos. Os níveis de C3 e microglobulina $\beta_{2}$ não apresentaram alterações significativas nos grupos, enquanto que o TNF $\alpha$ estava em altos níveis nos grupos com IRA hemodialisados com polissulfona e acetato de celulose, sendo a detecção maior neste último. Não ocorreram alterações significativas relacionadas com a pressão arterial sistêmica e outros parâmetros hematológicos. Pode-se concluir que houve aumento
\end{abstract}


nos níveis de TNF $\alpha$ nos cães com IRA submetidos à HD, e que a membrana de polissulfona se mostrou ligeiramente mais biocompatível que a membrana de acetato de celulose, porém novos marcadores precisam ser estudados.

Palavras-chave: Hemodiálise experimental; Cães; Insuficiência renal aguda; Biocompatibilidade; Hemodialisador.

\begin{abstract}
The dialysis procedures as hemodialysis (HD) try to keep the patient with acute kidney injury (AKI) alive until kidney regeneration. This paper evaluates the biocompatibility of cellulose acetate and polysulfone membranes in healthy dogs and in AIK ones submited to hemodialyses, analyzing biological parameters and clinical signs presented in this procedure. The hemodialysis animals were divided in four groups (each one with 8 dogs): group 1 composed by healthy animals that used cellulose acetate membrane; group 2 constituted by dogs with AKI that used cellulose acetate membrane; group 3 formed by healthy animals that used polysulfone membrane; group 4 composed by dogs with AKI that used polysulfone membrane. AKI induction was made by applying gentamicin (45mg/kg/EV/day). The main change was related to weight loss of the animals in the groups with AKI. There was also a significant reduction in urea and creatinine levels, mainly in hemodialysis animals with polysulfone membrane, while sodium, potassium and glucose levels did not show significant differences between the groups. The C3's and microglobulin $\beta_{2}$ levels didn't present significant changes in the groups, while the TNF $\alpha$ was in high levels in AKI's groups hemodialysed with polysulfone and cellulose acetate membranes, with higher level found in the last one. It didn't have significant changes related to arterial blood pressure and others hematologial parameters. It is possible conclude that there was an increase in TNF $\alpha$ level from AKI's dogs submitted to hemodialyses, and polysulfone membrane was slightly more biocompatible than cellulose acetate membrane, however more markers should be tested.
\end{abstract}

Keywords: Experimental hemodialyses; Dogs, Acute kidney injury; Biocompatibility; Hemodialyser.

\title{
Resumen
}

Los procedimientos de diálisis como la hemodiálisis (HD) buscan mantener la vida de los pacientes con insuficiencia renal aguda (IRA), hasta que se recupere la función renal. Este trabajo tiene como objetivo evaluar la biocompatibilidad de membranas de acetato de celulosa y polisulfona en perros sin y con IRA sometidos a hemodiálisis, a través de las respuestas biológicas y los signos clínicos presentes en el proceso. Los animales de hemodiálisis se subdividieron en cuatro grupos ( 8 animales en cada uno): grupo 1 compuesto por animales normales que utilizaron una membrana de acetato de celulosa; grupo 2 formado por animales con IRA que utilizaron acetato de celulosa; grupo 3 compuesto por animales normales que utilizaron membrana de polisulfona; y grupo 4 formado por animales con IRA que utilizaron polisulfona. La inducción de IRA se realizó mediante la administración de gentamicina (45 mg/kg / EV / día). Hubo una reducción significativa en los niveles de urea y creatinina, principalmente en animales en hemodiálisis con membrana de polisulfona, mientras que los niveles de sodio, potasio y glucosa no mostraron diferencias significativas entre grupos. Los niveles de C3 y microglobulina $\beta_{2}$ no mostraron cambios significativos en los grupos, mientras que TNF $\alpha$ estuvo en niveles elevados en los grupos con IRA hemodializados con polisulfona y acetato de celulosa, con la mayor detección en estos últimos. No hubo cambios significativos relacionados con la presión arterial sistémica y otros parámetros hematológicos. Se puede concluir que hubo un aumento en los niveles de TNF $\alpha$ en perros con IRA sometidos a HD, y que la membrana de polisulfona era ligeramente más biocompatible que la membrana de acetato de celulosa, sin embargo es necesario estudiar nuevos marcadores.

Palabras clave: Hemodiálisis experimental; Perros; Insuficiencia renal aguda; Biocompatibilidad; Hemodiálisis.

\section{Introdução}

A Insuficiência Renal Aguda (IRA) é uma síndrome clínica caracterizada pela diminuição súbita da filtração glomerular com subsequente acúmulo de toxinas metabólicas e perda da regulação do equilíbrio hídrico, eletrolítico e ácidobásico (Cowgill \& Elliot, 2004). Na Medicina Veterinária, a IRA ocorre de maneira relativamente frequente, desordens prérenais, renais intrínsecas e pós-renais podem causar IRA em cães. Instabilidade hemodinâmica e diminuição do débito cardíaco são as principais causas pré-renais que levam ao estabelecimento da IRA. Quando a diminuição de perfusão renal se perpetua, a situação isquêmica pode causar necrose tubular aguda (NTA), que também pode ocorrer pela ação de substâncias ou drogas nefrotóxicas, dentre elas antibióticos aminoglicosídeos, como a gentamicina (Flemenbum, 1973). 
A hemodiálise (HD) é o método que utiliza "rim artificial" para corrigir os desequilíbrios de composição e volume do fluido corpóreo, e eliminar toxinas acumuladas. Para que seja realizada, o sangue do paciente entra em contato com a membrana semipermeável e esta permitirá trocas com a solução dialisante (Cowgill \& Langstone, 1996).

Os hemodialisadores podem ser classificados de acordo com o tipo de membrana. As celulósicas são membranas polissacarídicas obtidas de algodão prensado, compostas de anéis com grupos hidroxila livres abundantes, que recebem vários nomes, como celulose regenerada, celulose cuprammonium, rayon cuprammonium e celulose ester saponificada. As de celulose regenerada são obtidas através da ligação química de material ao grupo hidroxila livre na superfície do polímero celulósico, sendo a mais comum de acetato de celulose, no qual o acetato substitui cerca de $80 \%$ dos grupos hidroxila, além das membranas diacetato e triacetato de celulose (Daugirdas et al., 2003).

As membranas de celulose substituída são modificadas através da adição de material sintético (dietilaminoetil) à celulose liquefeita, durante a sua fabricação. Como resultado, a superfície da membrana é alterada aumentando a biocompatibilidade. Existem ainda as membranas sintéticas que não são baseadas em celulose, e os materiais usados incluem poliacrilonitrila (PAN), polissulfona, policarbonato, poliamida e polimetilmetracrilato (PMMA). Possui baixa capacidade de ativação do sistema complemento (Daugirdas et al., 2003).

São diversas as respostas biológicas produzidas pelas membranas dos dialisadores (Hansen, 1991). As consequências biológicas da interação entre sangue e dialisador na IRA são mais inferenciais do que definitivas. Teoricamente, as membranas bioincompatíveis podem piorar o estado catabólico da IRA e agravar o estado inflamatório na sepse, condição comum nesse tipo de condição clínica. Além disso, a ativação e subsequente exaustão das células mono e polimorfonucleares pode predispor os pacientes a infecções (Haag-Webwr, 1996). Segundo Basile e Druke (1989), as principais alterações observadas durante o procedimento hemodialítico são trombocitopenia transitória, leucopenia e neutropenia, hipoxemia, síndrome do primeiro uso, amiloidose e liberação de citocinas como IL-1 e TNF.

A realização da presente pesquisa justifica-se pela necessidade de se obter respostas referentes à biocompatibilidade das membranas dos dialisadores, já que a literatura é escassa no que se refere ao assunto em questão, especialmente na IRA. Além disso, no Brasil, não existem descrições desse tipo de trabalho, do ponto de vista veterinário, o que pode trazer benefícios não só para a comunidade veterinária, mas também para a aplicação em medicina humana.

\section{Metodologia}

A pesquisa foi realizada no Laboratório Experimental do Departamento de Clínica Médica da Faculdade de Medicina de Botucatu - UNESP, Campus de Botucatu, com aprovação do Comitê de Ética em Pesquisa Animal da Faculdade de Medicina - UNESP, Campus de Botucatu (Protocolo n 321, 07/07/2003).

Foram utilizados 32 cães, sem raça definida, adultos, machos, sendo 16 clinicamente sadios e 16 com IRA induzida por gentamicina, com peso corporal variando entre 07 a $14 \mathrm{Kg}$, provenientes do Canil do Biotério Central da UNESP- Campus de Botucatu. Os animais foram divididos em quatro grupos, a saber:

- Grupo 1: constituído por 08 animais, clinicamente sadios, que receberam tratamento hemodialítico, utilizando membrana de acetato de celulose, a cada 24 horas, totalizando cinco sessões de HD.

- Grupo 2: constituído por 08 animais que, após estabelecimento da IRA, receberam tratamento hemodialítico, utilizando membrana de acetato de celulose, a cada 24 horas, totalizando cinco sessões de HD.

- Grupo 3: constituído por 08 animais, clinicamente sadios, que receberam tratamento hemodialítico, utilizando membrana de polissulfona, a cada 24 horas, totalizando cinco sessões de HD.

- Grupo 4: constituído por 08 animais que, após estabelecimento da IRA, receberam tratamento hemodialítico, utilizando membrana de polissulfona, a cada 24 horas, totalizando cinco sessões de HD. 
A gentamicina foi administrada na dose de $15 \mathrm{mg} / \mathrm{Kg}$, dividida em aplicações a cada 8 horas, via intravenosa. Antes da administração do fármaco e a cada 24 horas após seu início, foram realizadas dosagens séricas de ureia, creatinina, sódio e potássio. As coletas de sangue venoso foram por punção da veia jugular externa. O estabelecimento da IRA foi definido quando os animais apresentassem concentração sérica de creatinina $\geq 5 \mathrm{mg} / \mathrm{dL}$, independente do volume urinário.

Os animais foram submetidos à implantação do cateter de duplo lúmen (8 French), preferencialmente na veia jugular externa direita, segundo técnica descrita por MENESES et. al., (2002),. Em seguida foi realizada fluoroscopia cérvico-torácica na posição látero-lateral e dorso-ventral, para verificação do correto posicionamento do cateter (veia cava cranial ou átrio direito). Os animais foram anestesiados utilizando-se propofol (6,6 - 8,8 $\mathrm{mg} / \mathrm{Kg}$ IV in bolus), sendo o tempo de anestesia aproximadamente 10 minutos.

Os cães também foram submetidos a exame físico completo, antes e após as sessões de HD, de acordo com Mccurnin e Poffenbager (1991), constando de:

- Avaliação do estado de hidratação antes e após as sessões de HD;

- Avaliação dos linfonodos (sub-mandibulares, pré-escapulares, poplíteos), quanto a tamanho, consistência e mobilidade (pré e pós-HD);

- Avaliação da coloração das mucosas aparentes (pré e pós-HD);

- Avaliação do tempo de preenchimento capilar (pré e pós-HD);

- Auscultação torácica (pulmões e coração) (pré, 5', 30' e pós-HD);

- Palpação abdominal (pré e pós-HD);

- Verificação da temperatura corporal do animal (pré, 5', 30' e pós-HD).

- Todos os animais foram pesados, antes e depois de cada sessão de HD, utilizando-se balança digital (Filizola ${ }^{\circledR}$ ).

- Verificação da pressão arterial sistêmica (sistólica) pelo aparelho DV-10 ${ }^{\circledR}$, utilizando-se o método não-invasivo, através do Doppler vascular nos momentos pré, 5, 30 e pós HD.

Foram colhidas amostras de sangue para realização de dosagens de ureia $(\mathrm{mg} / \mathrm{dL})$, creatinina $(\mathrm{mg} / \mathrm{dL})$, sódio (mmol/L), potássio (mmol/L) e glicose (g/\%), antes e após a realização das sessões de HD.

A biocompatibilidade da membrana do dialisador foi avaliada através da contagem do número de leucócitos (células / $\mu \mathrm{L}$ ) e diferencial (\%), e contagem global de plaquetas (células/ $\mu \mathrm{L}$ ) nos momentos pré e pós HD. Determinação do nível sérico de $\mathrm{C} 3$ e microglobulina $\beta_{2}$ utilizando-se kits convencionais nos momentos pré e pós HD da primeira, terceira e quinta sessões de HD. A detecção de TNF- $\alpha$ foi realizada através de ensaio biológico e leitura através de ELISA, nos momentos pré, 5, 30 e pós HD.

Para a avaliação da segurança do método dialítico em busca de identificar episódios hipotensivos (PAS < $80 \mathrm{mmHg}$ ), para tanto verificou-se a pressão arterial sistêmica (sistólica) pelo aparelho DV-10 ${ }^{\circledR}$, utilizando-se o método não-invasivo, através do Doppler vascular, cujo transdutor foi colocado, preferencialmente, na artéria mediana, e manguito, cuja escolha de tamanho levou em consideração $40 \%$ do diâmetro do membro, no terço médio da pata utilizada, nos momentos pré, 5, 30 e pós HD. Outro item avaliado foi à percentagem de animais com necessidade transfusional (volume globular $<15 \%$ ), verificando-se o número de hemácias (células/ $\mu \mathrm{L}$ ), hemoglobina (g/dL), e volume globular, antes e após as sessões de HD.

O tempo de coagulação ativada (TCA) foi avaliado antes, com 5, 30 e 60 minutos de HD. Este teste é semelhante ao tempo de coagulação ativado de Lee White, utilizando-se um ativador (pó de vidro), que é adicionado ao tubo de ensaio para acelerar a coagulação. Para determinação do TCA, $2 \mathrm{~mL}$ de sangue foram adicionados ao tubo de vidro próprio e submetido à leitura no monitor (MCA $2000^{\circledR}$ Adib Jatene/SP). O resultado deste teste é apresentado em segundos (zero a 2000).

Avaliou-se também a percentagem de animais que evoluíram para óbito durante o experimento. 
Para a realização da HD foi utilizado aparelho de HD de sistema proporcional (System 1000® Series - Tina - Baxter Co.), com módulo de ultrafiltração controlada. O circuito extracorpóreo foi conectado como descrito por COWGILL (1995). Os animais receberam "priming” com solução fisiológica 0,9\% para que se evitasse quadro hipovolêmico e hipotensivo ao início das sessões. O volume administrado foi de aproximadamente 180mL, conforme descrito por MENESES, (2003). O fluxo sanguíneo extracorpóreo foi restrito a 3-5 mL/Kg/min nos dois tratamentos iniciais, evitando excessivo clearance de uréia e desequilíbrio osmótico. A partir da terceira sessão ocorreu aumento progressivo para $10-15 \mathrm{~mL} / \mathrm{Kg} / \mathrm{min}$. As sessões foram programadas com duração de 60 minutos, com intervalo de 24 horas, totalizando cinco sessões para cada animal.

Neste experimento utilizou-se taxa de UF estabelecida pela necessidade de remoção de fluido, pela elevação do peso dos animais no período interdialítico e pelo volume de "priming” infundido.

A solução de diálise foi utilizada solução tampão de bicarbonato de sódio (Solução bicarbonatada 8,4\% ${ }^{\circledR}$ - Farmarin). O fluxo do dialisato (Concentrado polietletrolítico para $H D^{\circledR}$ - Farmarin) foi de $500 \mathrm{~mL} /$ minuto. O pH desta solução varia entre 6,8 e 7,2 e a osmolaridade é de 206 mOsm.

As membranas utilizadas foram de polissulfona (Hemoflow F3® - Fresenius polysulfone - Fresenius Medical Care) e acetato de celulose (Cellulose Acetate Hollow Fiber Dialyzer ${ }^{\circledR}$ - Baxter Healthcare Corporation), com áreas de superfície de 0.4 e $0.5 \mathrm{~m}^{2}$, respectivamente.

Neste experimento, optou-se pela utilização de perfil de sódio para evitar quadro hiponatrêmico e hipotensivo e suas conseqüências, tornando as sessões de HD seguras. Utilizou-se durante os 10 minutos iniciais $155 \mathrm{mmol} / \mathrm{L} ; 150 \mathrm{mmol} / \mathrm{L}$ nos 25 minutos intermediários e $145 \mathrm{mmol} / \mathrm{L}$, nos 25 minutos subseqüentes, adaptando o perfil descrito por MENESES, (2003).

A anticoagulação foi realizada com heparina sódica (Liquemine ${ }^{\circledR}$ - 25.000 U.I. / 5 mL - Roche) em protocolo intermitente com dose inicial de 100U / Kg, conforme descrito por BRANT, (2003). Antes da sessão, 5, 30 e 60 minutos após o início do procedimento hemodialítico, foi determinado o TCA. Doses suplementares de $25 \mathrm{U} / \mathrm{Kg}$ seriam administradas aos 30 minutos do início do procedimento ou quando o TCA fosse menor que 1,5 vezes o valor inicial.

Imediatamente após a realização das sessões de HD, coletou-se sangue para realização dos exames laboratoriais (hematológicos, bioquímicos, e testes de biocompatibilidade), e para tal utilizou-se o cateter de duplo lúmen instalado na veia jugular externa. Em seguida, era realizada anti-sepsia do orifício de saída do cateter e das vias arterial e venosa do cateter. Posteriormente as vias eram lavadas com solução fisiológica $0,9 \%$, e preenchidas com heparina sódica $(0,7 \mathrm{~mL}$ da via arterial e 0,8 mL da via venosa) para evitar a presença de coágulos nas vias, cuja presença diminuiria a perviedade do cateter e até poderia impossibilitar sua utilização. Foi colocado curativo compressivo no local para evitar que os animais retirassem acidentalmente o cateter.

\subsection{Análise estatística}

Para as variáveis do exame físico, pressão arterial, tempo de coagulação ativada e avaliação da biocompatibilidade da membrana do dialisador foi realizada técnica da análise de variância (paramétrica ou não paramétrica) para o esquema de dois fatores (grupo quanto a presença ou não da doença e grupo quanto ao tipo de membrana utilizada), completada com os respectivos testes de comparações múltiplas (Norman \& Treiner, 1994).

O conjunto de dados foi submetido ao modelo multinível, utilizando três níveis de análise: no primeiro se realizou a comparação entre todos os animais do experimento e as cinco sessões de HD; no segundo a comparação levando em consideração o tipo de dialisador utilizado (acetato de celulose ou polissulfona) e no terceiro a comparação levando em consideração a condição clínica dos animais, ou seja, se estavam em IRA ou não. As análises foram feitas pelo PROC MIXED do SAS, v. 8.02. Todos as discussões foram realizadas no nível de 5\% de significância. 


\section{Resultados}

Os dados são referentes aos momentos pré (pré-HD) e pós-hemodiálise (pós-HD) (dados clínicos, valores hematológicos e bioquímicos) e momentos pré (M0), 5' (M5), 3’ (M30), e 60' (M60) de HD para avaliação imunológica, TCA e PA das cinco sessões realizadas em cada animal.

Nos exames físicos realizados pré e pós-hemodiálise dos grupos 1 e 3, não foram encontradas alterações significativas relacionadas ao estado de hidratação, linfonodos, auscultação pulmonar e torácica, e palpação abdominal. Nos grupos 2 e 4 a principal alteração estava relacionada com o estado de hidratação, onde a grande maioria encontrava-se em diferentes graus de desidratação.

Em relação à coloração das mucosas aparentes, verificou-se que, no momento pré-hemodiálise as mesmas encontravam-se normocoradas, e no momento pós-hemodiálise ligeiramente hipocoradas, em alguns indivíduos de todos os grupos estudados.

Os resultados relativos à temperatura retal $\left(\mathrm{T}^{\circ} \mathrm{C}\right)$ acham-se expressos em média nos momentos $\mathrm{MO}, \mathrm{M} 5, \mathrm{M} 30$ e M60 dos grupos 1, 2, $3 \mathrm{e}$ 4, respectivamente. As médias de $\mathrm{T}^{\circ} \mathrm{C}$ do grupo 1 foram de 38,73 \pm 0,45 em MO, 38,6 \pm 0,47 em M5, 38,32 \pm 0,47 em M30, e 38,6 \pm 0,41 em M60. Do grupo 2 foram de 37,4 \pm 1,02 em MO, 37,24 $\pm 0,93 \mathrm{em} \mathrm{M5,} \mathrm{37,18} \pm 0,68 \mathrm{em}$ M30, e 37,21 \pm 0,73 em M60. No grupo 3 as médias foram de 38,29 \pm 0,35 em MO, 37,98 \pm 0,49 em M5, 37,75 $\pm 0,39 \mathrm{em}$ M30, e 37,8 \pm 0,34 em M60. No grupo 4 foram de 38,04 \pm 0,37 em MO, 37,93 \pm 0,44 em M5, 37,82 $\pm 0,39$ em M30, e 37,84 \pm 0,38 em M60. Não houve diferença estatisticamente significante entre os grupos estudados $(\mathrm{P}<0,005)$.

Quanto ao tempo de preenchimento capilar, no momento pré-hemodiálise apresentava-se em um segundo (1“) e póshemodiálise em 2 segundos (2"), em todos os grupos estudados.

Os resultados referentes à contagem de hemácias (células $/ \mu \mathrm{L})$, hemoglobina $(\mathrm{g} / \mathrm{dL})$, volume globular $(\%)$, contagem de plaquetas (células $/ \mu \mathrm{L}$ ), contagem de leucócitos (células/ $\mu \mathrm{L}$ ) e diferencial (\%), nos momentos pré-HD e pós-HD dos grupos 1, 2, 3 e 4, acham-se expressos em média.

Em relação a contagem global de hemácias as médias nos momentos pré-HD e pós-HD do grupo 1 foram, respectivamente, 5,58 \pm 0,43 e 4,88 \pm 0,9. Do grupo 2 4,32 $\pm 1,09$ e 4,04 $\pm 1,35$. Do grupo $34,78 \pm 1,01$ e 4,08 $\pm 1,05$, e do grupo $44,57 \pm 1,24$ e 4,27 $\pm 1,21$. A análise estatística não mostrou diferença significativa quando se avaliou os três níveis do modelo $(\mathrm{P}>0,05)$.

Os resultados referentes aos momentos pré-HD e pós-HD do volume globular dos grupos 1, 2, 3 e 4 foram, respectivamente, $36,53 \pm 5,85$ e $32,08 \pm 6,51 ; 29,35 \pm 7,23$ e $26,55 \pm 7,63 ; 32,92 \pm 6,16$ e $28,01 \pm 6,44 ;$ e $31,56 \pm 8,88$ e 29,83 $\pm 9,27$. Estatisticamente não houve diferença significativa quando se avaliou o primeiro e segundo níveis $(\mathrm{P}>0,05)$, porém quando se avaliou o terceiro nível, houve diferença $(\mathrm{P}<0,05)$.

Os valores das médias dos momentos pré-HD e pós-HD de hemoglobina foram, respectivamente, nos grupos 1, 2, $3 \mathrm{e}$ 4 de 12,21 $\pm 1,98$ e 10,83 $\pm 2,09 ; 9,75 \pm 2,37$ e $8,89 \pm 2,67 ; 11,08 \pm 2,22$ e 9,49 $\pm 2,26$; e 10,76 $\pm 3,18$ e 9,97 $\pm 3,32$. A análise estatística não mostrou diferença significativa quando se avaliou os três níveis do modelo $(\mathrm{P}<0,005)$.

As médias do número total de plaquetas dos grupos 1, 2, 3 e 4 foram, respectivamente, $66 \pm 24,7$ e 39,12 $\pm 14,66$; $173,25 \pm 80,12$ e 118,1 $\pm 63,1 ; 171,82 \pm 49,24$ e 119,15 $\pm 42,64 ;$ e 186,05 \pm 74,93 e 135,05 $\pm 61,34$, referentes aos momentos pré-HD e pós-HD. A análise estatística não mostrou diferença significativa quando se avaliou os três níveis do modelo $(\mathrm{P}<$ $0,05)$.

As médias do número de leucócitos nos momentos pré-HD e pós-HD dos grupos 1, 2, 3 e 4, foram, respectivamente, $14,31 \pm 3,42$ e $7,05 \pm 2,11 ; 12,27 \pm 7,23$ e $8,76 \pm 4,93 ; 11,41 \pm 4,18$ e 7,21 $\pm 3,22$; e $12,63 \pm 5,73$ e $9,09 \pm 4,4$. Estatisticamente 
não houve diferença significativa quando se avaliou o primeiro e segundo níveis ( $\mathrm{P}>0,05)$, porém quando avaliou-se o terceiro houve diferença $(\mathrm{P}<0,05)$.

Em relação a contagem diferencial de leucócitos, as médias do número de neutrófilos nos momentos pré-HD e pósHD dos grupos 1, 2, 3 e 4 foram, respectivamente, 73,19 \pm 13,57 e 74,42 \pm 8,7; 73,3 $\pm 18,42$ e 73,25 $\pm 17,17 ; 69,59 \pm$ 7,33 e $72,85 \pm 8,97$; e 69,82 $\pm 16,62$ e 69,96 \pm 18,34. A análise estatística não mostrou diferença significativa quando se os três níveis do modelo $(\mathrm{P}>0,05)$.

Quanto aos linfócitos, as médias foram, respectivamente, $21,75 \pm 12,61$ e $23,17 \pm 8,24 ; 23,57 \pm 17,63$ e $24,19 \pm$ 16,$72 ; 22,56 \pm 7,53$ e $21,59 \pm 9,15$; e $27,21 \pm 12,43$ e $27 \pm 14,73$. A análise estatística não mostrou diferença significativa quando se avaliou três níveis do modelo $(\mathrm{P}>0,05)$.

As médias dos monócitos foram, respectivamente, 4,21 $\pm 2,41$ e 2,22 $\pm 0,99 ; 2,57 \pm 1,73$ e 2,08 $\pm 1,48 ; 6,99 \pm 4,92$ e $4,95 \pm 3,14$; e 1,86 $\pm 1,84$ e 1,77 $\pm 1,92$. A análise estatística não mostrou diferença significativa quando se avaliou os três níveis do modelo $(\mathrm{P}<0,05)$.

Os eosinófilos tiveram médias, respectivamente, de $0,4 \pm 0,36$ e $0,2 \pm 0,15 ; 0,39 \pm 0,4$ e $0,37 \pm 0,38 ; 0,36 \pm 0,2$ e 0,36 $\pm 0,58$; e $0,12 \pm 0,18$ e $0,12 \pm 0,2$. A análise estatística não mostrou diferença significativa quando se avaliou os três níveis do modelo $(\mathrm{P}<0,05)$.

As médias dos basófilos foram, respectivamente, de $0,4 \pm 0,36$ e $0,21 \pm 0,12 ; 0,14 \pm 0,12$ e $0,1 \pm 0,06 ; 0,21 \pm 0,13$ e $0,15 \pm 0,08$; e $0,05 \pm 0,07$ e $0,04 \pm 0,06$. Estatisticamente não houve diferença significativa quando se avaliou os dois primeiros níveis $(\mathrm{P}>0,05)$, porém quando se avaliou o terceiro nível do modelo houve diferença $(\mathrm{P}<0,05)$.

Os resultados relativos às determinações de tempo de TCA (segundos) nos momentos M0, M5, M30 e M60 dos grupos 1, 2, 3 e 4 estão expressos em média.

No grupo 1 as médias foram, respectivamente, de 90,62 \pm 14,17 em MO, 287,32 \pm 147,45 em M5, 192,15 $\pm 53,28 \mathrm{em}$ M30, e 125,57 \pm 26,13 em M60. Do grupo 2 foram de 88,62 \pm 23,78 em MO, 360,3 \pm 305 em M5, 305,77 \pm 308,43 em M30, e 238,25 \pm 223,56 em M60. No grupo 3 as médias foram de 84,25 \pm 34,31 em MO, 310,97 \pm 154,79 em M5, 219,17 \pm 88,69 em M30, e 145,77 \pm 39,14 em M60. No grupo 4 foram de 131,62 \pm 49,53 em MO, 408,7 \pm 226,67 em M5, 275,7 \pm 123,05 em M30, e 161,87 \pm 51,86 em M60. Houve diferença entre os momentos M0 e M5, M30 e M60, enquanto que não houve diferença estatisticamente significativa entre os momentos M5, M30 e M60 de todos os grupos $(\mathrm{P}<0,005)$.

Em relação ao volume de preenchimento dos dialisadores de polissulfona $(28 \mathrm{~mL})$ e acetato de celulose utilizados (34 $\mathrm{mL}$ ) e o reuso, observou-se que a média foi de $23 \pm 4,28$ para o primeiro dialisador, enquanto que o segundo não permitiu a reutilização provocando descarte após seu uso.

Os valores das médias de ureia nos momentos pré-HD e pós-HD dos grupos 1, 2, 3 e 4 foram, respectivamente, $28,8 \pm$ 8,6 e $20,25 \pm 5,02 ; 205,2 \pm 72,88$ e $147,8 \pm 51,45 ; 31,27 \pm 9,04$ e $18,72 \pm 7,05$; e $214,62 \pm 49,18$ e $121,12 \pm 38,41$. Estatisticamente, quando se utilizou o modelo multinível, analisando-se o primeiro e segundo níveis houve diferença estatisticamente significativa $(\mathrm{P}<0,05)$ enquanto que ao analisar-se o terceiro nível não houve diferença significativa.

Os valores das médias de creatinina referentes aos momentos pré-HD e pós-HD dos grupos 1, 2, 3 e 4 que foram, respectivamente, $28,8 \pm 8,6$ e 20,25 $\pm 5,02 ; 205,2 \pm 72,88$ e $147,8 \pm 51,45 ; 31,27 \pm 9,04$ e 18,72 \pm 7,05; e 214,62 $\pm 49,18$ e $121,12 \pm 38,41$. A análise estatística mostrou diferença significativa quando se avaliou o primeiro e terceiro níveis do modelo $(\mathrm{P}<0,05)$, enquanto que ao avaliar-se o segundo nível, não houve diferença significativa $(\mathrm{P}>0,05)$. 
Os valores médios de sódio foram $147,72 \pm 2,05$ e 146,25 $\pm 2,25 ; 145,15 \pm 5,31$ e 143,87 $\pm 5,01 ; 146,87 \pm 4,17$ e 146,67 \pm 2,29; e 148,85 \pm 14,45 e 145,22 \pm 3,98, referentes, respectivamente, aos momentos pré-HD e pós-HD, dos grupos 1, 2, 3 e 4. A análise estatística não mostrou diferença significativa quando se avaliou os três níveis do modelo $(\mathrm{P}>0,05)$.

Os valores das médias de potássio referentes aos momentos pré-HD e pós-HD dos grupos 1, 2, 3 e 4 que foram, respectivamente, $3,71 \pm 0,41$ e $3,26 \pm 0,29 ; 3,87 \pm 0,41$ e $3,34 \pm 0,28 ; 3,94 \pm 0,16$ e 3,11 $\pm 0,35 ;$ e $3,89 \pm 0,67$ e 3,27 $\pm 0,72$. A análise estatística não mostrou diferença significativa quando se avaliou os três níveis do modelo $(\mathrm{P}>0,05)$.

Os valores das médias de glicose $(\mathrm{g} \%)$ nos momentos pré-HD e pós-HD dos grupos 1, 2, 3 e 4 foram, respectivamente, $103,02 \pm 5$ e 95,77 \pm 9,61; 95,3 \pm 9,03 e 82,55 $\pm 11,79 ; 128,82 \pm 25,25$ e 102,4 $\pm 22,24$; e 112,12 $\pm 14,19$ e $88,17 \pm 21$. A análise estatística não mostrou diferença significativa quando se avaliou os três níveis do modelo $(\mathrm{P}>0,05)$.

Os resultados relativos à pressão arterial sistólica (PAS) em mmHg, referentes aos momentos M0, M5, M30, e M60 (resultados em média), no grupo 1 as médias foram, respectivamente, de 107,75 \pm 10,97 em MO, $130,75 \pm 12,06$ em M5, $116 \pm$ 13,35 em M30, e 102,75 \pm 10,85 em M60. Do grupo 2 foram de 158,75 \pm 33,29 em MO, 148,25 \pm 31,93 em M5, 134,25 \pm 37,13 em M30, e 146,12 \pm 33,02 em M60. No grupo 3 as médias foram de 111,75 $\pm 13,93$ em MO, $90 \pm 15,48$ em M5, 97,12 \pm 12,18 em M30, e 96,5 \pm 11,66 em M60. No grupo 4 foram de $152 \pm$ 30,73 em MO, 165,5 \pm 36,3 em M5, 145 \pm 39,28 em M30, e 140,4 \pm 36,06 em M60. Não houve diferença estatisticamente significante entre os grupos estudados (P> $0,05)$.

A avaliação da biocompatibilidade das membranas dos dialisadores de polissulfona e acetato de celulose foram realizadas avaliando-se nos momentos pré-HD e pós-HD o nível sérico de microglobulina $\beta_{2}$ e detecção de TNF- $\alpha$ nos momentos MO, M5, M30 e M60. Os resultados foram expressos em média.

Em relação à microglobulina $\beta_{2}$ os valores pré-HD e pós-HD foram $0,0 \mathrm{mg} / \mathrm{L}$, enquanto que os valores de $\mathrm{C} 3$ foram $<15,6 \mathrm{mg} / \mathrm{dL}$. Não houve diferença estatisticamente significativa quando se avaliou C3 e microglobulina $\beta_{2}(\mathrm{P}>0,05)$.

Quanto ao TNF- $\alpha$, as médias nos momentos M0, M5, M30 e M60 dos grupos 1, 2, 3 e 4 foram, respectivamente, 0 em MO, M5, M30, e M60 do grupo 1. Do grupo 2 foram de 434,9 em MO, 572,03 em M5, 719,78 em M30, e 545,59 em M60. No grupo 3 as médias foram de 0 em MO, M5, M30, e M60. No grupo 4 foram de 523,95 em MO, 369,8 em M5, 389,89 em M30, e 225,28 em M60. Os resultados são expressos em pg/mL. Não houve diferença estatisticamente significativa entre os grupos estudados $(\mathrm{P}>0,05)$.

Ainda, vale ressaltar que não ocorreu óbito durante a realização deste experimento.

\section{Discussão}

Na Medicina Veterinária, a IRA é uma das maiores causas de morte em cães e gatos, visto que o tratamento rotineiramente utilizado, baseado em fluidoterapia e tentativa de reversão de oligúria, caso esta esteja presente no quadro, nem sempre é eficiente. A terapia renal substitutiva, seja diálise peritoneal (DP) ou hemodiálise (HD), tem se mostrado alternativa viável para o tratamento de cães e gatos com IRA. A DP tem algumas complicações na Medicina Veterinária, como a manutenção do acesso peritoneal, o que dificulta e limita sua utilização na rotina clínica. A HD, por sua vez, vem sendo desmistificada e sua aplicação clínica se torna cada vez mais presente no dia a dia do médico veterinário.

Este trabalho foi desenvolvido, haja vista a necessidade de se estudar em cães a biocompatibilidade de duas membranas, no caso foi testada as membranas de acetato de celulose e polissulfona, utilizada rotineiramente em pacientes humanos com IRA. Nas últimas décadas as pesquisas no campo da hemodiálise têm se caracterizado por aumento na atenção a respeito de várias reações químicas e biológicas, além dos efeitos colaterais resultantes da interação entre o sangue do paciente 
e os materiais utilizados durante o procedimento hemodialítico, particularmente as membranas dos dialisadores, fenômeno este denominado de biocompatibilidade, como descrito por Basile e Drueke (1989).

Neste estudo diversos aspectos foram abordados para se avaliar a biocompatibilidade das membranas dos dialisadores de acetato de celulose e polissulfona em cães. Dentre eles podemos destacar as alterações clínicas, hematológicas e bioquímicas, complicações decorrentes da realização do procedimento, como hipotensão, necessidade transfusional e ocorrência de óbitos, e também as respostas imunológicas como produção de TNF $\alpha, \mathrm{C} 3$ e microglobulina $\beta_{2}$.

Os exames bioquímicos não revelaram alterações importantes durante e após a HD nos animais normais, diferentemente do ocorrido naqueles em IRA. Em relação ao sódio não ocorreram mudanças importantes no nível sérico deste eletrólito em todos os grupos estudados, possivelmente decorrente da utilização do chamado perfil de sódio, indicado por Cowgill, (1996) e descrito por Meneses, (2003), cuja finalidade é não permitir que os níveis deste elemento diminuam durante a realização do procedimento hemodialítico, evitando-se assim a ocorrência de hiponatremia e suas consequências como hipotensão, náusea, emese, dentre outras.

Os dados relativos às concentrações pré e pós-dialíticas de ureia mostraram queda significante, tanto nos animais sadios quanto nos doentes, sendo o valor pós dialítico, aproximadamente 45\% do valor inicial. Esta redução é compatível com clearance adequado dessa molécula, em que pese à concentração sanguínea anterior à diálise, que ressalta eficácia do método hemodialítico na remoção de toxinas de pequeno peso molecular, independentemente do tipo de membrana utilizada. Os níveis séricos de creatinina também foram menores quando se comparou os momentos pré e pós-hemodiálise em todos os grupos estudados, principalmente nos animais com IRA, fato este que confirma o êxito na realização do procedimento.

A utilização de banhos isentos de glicose é comum na rotina clínica de centros de HD. Há possibilidade, com a utilização deste tipo de banho, da ocorrência de hipoglicemia, o que não foi observado no presente estudo. Este acontecimento deve ser decorrente de adequada neoglicogênese durante o procedimento hemodialítico, como proposto para pacientes humanos (Riella 1996). Os dados do presente trabalho confirmam o mesmo fenômeno em cães normais e com IRA, submetidos ao tratamento hemodialítico.

Não se observou a presença de leucopenia nos grupos estudados, assim como relatado por Basile \& Drueke, (1989), nem linfopenia e monocitose como citado por Amore \& Coppo, (2002). Há íntima correlação entre leucopenia e ativação do sistema complemento, fato este que não ocorreu no presente trabalho, haja vista que os níveis séricos de C3 permaneceram dentro da normalidade em todos os grupos estudados, justificando desta forma a resposta encontrada. Segundo Hoenich \& Stamp, (2000), o pico de detecção de C3 se dá aos 15 minutos de iniciado o procedimento hemodialítico, e aos 60 minutos o nível sérico tende a retornar ao basal, situação esta decorrente da resposta fisiológica da medula óssea à neutropenia periférica.

Em relação à microglobulina $\beta_{2}$, não foi possível a detecção do nível sérico desta substância durante a realização deste experimento, que pode ser creditado ao fato de que a mensuração se deu através do método de imunoturbidimetria, e não ELISA, assim como proposto por Nakajima et al., (1999) e Nakajima et al., (2000), além do que, a presença de altos níveis de microglobulina $\beta_{2}$ está intimamente correlacionada com a deposição de substância amilóide nas regiões articulares e periarticulares, particularmente em pacientes submetidos à HD crônica, assim como descrito por Basile \& Drueke, (1989).

Quanto à determinação do $\mathrm{TNF} \alpha$, pudemos notar que não houve detecção desta citocina nos animais normais, enquanto que nos animais doentes, verificamos a presença de altos níveis em ambas as membranas. Vale ressaltar, entretanto, que os níveis foram maiores no primeiro grupo.

Em pacientes submetidos à hemodiálise, a detecção de altos níveis de $\mathrm{TNF} \alpha$, citocina pró-inflamatória, podem estar correlacionados com um pior prognóstico dos pacientes com sepse e/ou com o desenvolvimento de disfunção orgânica múltipla, segundo Simmons et al., (2004). Geralmente, como descrito por Goldstein et al., (2003), o pico de produção se dá aos 
30 minutos de iniciado o procedimento e pode perdurar por até 24 horas, fato semelhante ao encontrado em nosso estudo, já que foram realizadas sessões diárias em todos os pacientes.

Pode-se também creditar os altos níveis desta citocina pelo fato dos animais estarem apresentando quadro de uremia importante, levando a um estado inflamatório persistente, fenômeno este denominado de "pré-ativação" dos monócitos, que leva este tipo celular a produzir grandes quantidades de TNF $\alpha$ sob estimulação constante, assim como descrito por Goldstein et al., (2003). Um ponto que deve ser enaltecido neste estudo é a correlação entre os altos níveis de TNFa e a presença de sinais clínicos importantes como inapetência, perda de peso e emagrecimento progressivo, além de episódios esporádicos de emese nos animais com IRA.

Como descrito por Singh et al., (2003), as membranas de celulose (acetato de celulose) apresentam em sua composição grupos hidroxil, que interagem com o sistema complemento levando a sua ativação, enquanto que as sintéticas (polissulfona) diferem das anteriores por apresentarem composição polimérica e hidrofobicidade, ocasionando menor capacidade de ativação do complemento e liberação de citocinas, assim como descrito neste trabalho.

Em relação a hematimetria, em todos os grupos estudados, pudemos notar que não houve alteração dos valores de hemácias, hemoglobina, com pequena diminuição do volume globular, fato diferente do relatado por Cowgill \& Langstone, (1996). Ocorrência esta, possivelmente explicada, pelo fato de que, durante a realização do procedimento hemodialítico, ocorreram, mesmo em pequenas quantidades, perdas sanguíneas, através das linhas e também dos dialisadores, ou de fenômenos hemolíticos, decorrentes da passagem do sangue pelo circuito extracorpóreo. Vale ressaltar que não houve necessidade transfusional em nenhum animal do experimento.

Com o protocolo de heparinização utilizado, conseguimos manter os valores de TCA dentro dos níveis adequados, evitando-se assim, nos animais do experimento, tanto a heparinização insuficiente quanto a excessiva, assim como descrito por MENESES, (2003). A obtenção da heparinização adequada permite múltiplos reusos dos dialisadores, importante para manter a viabilidade econômica da hemodiálise em animais e humanos. Neste estudo as membranas de acetato de celulose não foram reutilizadas, diferentemente do acontecido com as membranas de polissulfona.

Hansen (1991), indica algumas ações práticas para aumentar a biocompatibilidade do processo hemodialítico como utilizar dialisadores com área de superfície menor, fato este que acarretaria em menor exposição do sangue do paciente ao dialisador, selecionar individualmente para cada paciente a membrana a ser utilizada, e assegurar a pureza do dialisato, visto que a presença de contaminação pode levar a sintomas intradialíticos via ativação de monócitos e indução de citocinas.

Deve-se salientar que não ocorreu nenhum óbito durante a realização deste experimento, independentemente de serem normais ou com IRA, demonstrando a segurança do método e a possibilidade de reprodutibilidade do proposto por MENESES, (2003).

\section{Conclusão}

Os resultados obtidos no presente estudo permitiram concluir que ocorre aumento nos níveis séricos de TNF $\alpha$ nos animais com IRA submetidos a HD, refletindo resposta importante a produção de citocinas em ambas as membranas. Os hemodialisadores apresentam diferentes graus de biocompatibilidade, fato este que deve enaltecido no momento de se escolher por um determinado tipo de membrana do dialisador. Neste trabalho a membrana de acetato de celulose mostrou-se tão biocompatível quanto a de polissulfona, apesar desta última ter apresentado desempenho ligeiramente superior, não devendo, portanto, cair em desuso pelo fato de ser tarjada como bioincompatível. Um fator relevante na hora da escolha do tipo de membrana é o custo individual e o impacto relacionado com sua reutilização ou não. Assim, a biocompatibilidade das membranas dos dialisadores em cães permanece sem resolução, abrindo caminhos para o desenvolvimento de novas pesquisas 
Research, Society and Development, v. 10, n. 3, e15410312361, 2021

(CC BY 4.0) | ISSN 2525-3409 | DOI: http://dx.doi.org/10.33448/rsd-v10i3.12361

experimentais, utilizando outros marcadores como IL-1, IL-6, IL-18 e avaliação do estresse oxidativo, assim como de novos tipos de membranas.

\section{Referências}

Amore, A., \& Coppo, R. (2002). Immunological basis of inflammation in dialysis. Nephrol. Dial. Transplant. 17 (8), 16-24. https://doi.org/10.1007/s11255013-0460-9

Basile, C., \& Drueke, T. (1989). Dialysis membrane biocompatibility. Nephron. 52, 113-118. https://doi.org/10.1159/000185612

Brant, J. R. A. C. Estudo comparativo entre os anticoagulantes heparina sódica e heparina de baixo peso molecular em cães (Canis familiaris) submetidos à hemodiálise. 2003. 112p. Dissertação (Mestrado). Faculdade de Medicina Veterinária e Zootecnia, Universidade Estadual Paulista, Botucatu. Retrived from 01-CAPA-FINAL.doc (unesp.br)

Cowgill, L. D., \& Elliot, D. A. (2004). Tratado de Medicina Interna Veterinária: Insuficiência renal aguda. uanabara koogan.

Cowgill, L. D., \& Langston, C. E. (1996). Role of hemodialysis in the management of dogs and cats with renal failure. Veterinary Clinics of North America: Small Animal Practice. 26, 1347-78. https://doi.org/10.1016/S0195-5616(96)50132-4

Cowgill, L. D.; \& Maretzki, C. H. CVT update: Veterinary applications of hemodialysis. In: Bonagura, J. D., \& Kirk, R. W. (1995). Kirk's Current Veterinary Therapy XII: Small Animal Practice. Philadelphia: WB Saunders.

Daugirdas, J. T., Van Stone, J. C., \& Boag, J. T. (2003). Manual de Diálise: Aparelho de hemodiálise. MEDSI Editora Médica e Científica.

Flamenbaum, W. (1973). Pathophysiology of acute renal failure. Arch. Intern. Med. 10.1001/archinte.1973.00320120151011

Goldstein, S. L., Currier, H., Watters, L., et al. (2003). Acute and chronic inflammation in pediatric patients receiving hemodialysis. The J. Ped. 653-657. 10.1067/S0022-3476(03)00534-1.

Haag-Weber, M., \& Horl, W. H. (1996). Disfunction of polymorphonuclear leukocytes in uremia. Semin. Nephrol. 16, 192-201. Dysfunction of polymorphonuclear leukocytes in uremia - PubMed (nih.gov)

Hansen, S. K. (1991). Improving hemodialysis biocompatibility. Dial. Transpl. 20(10), 610-13.

Hoenich, N. A., \& Stamp, S. (2000). Clinical investigation of the role of membrane structure on blood contact and solute transport characteristics of a cellulose membrane. Biomat. 21, 317-324. Integrated Biomaterials Science | SpringerLink

Mccurnin, D. M.; \& Poffenbarger, E. M. (1991). Small animal phisical diagnosis and clinical procedures. Philadelphia: WB Saunders.

Meneses, A. M. C. (2003). Desenvolvimento de um modelo experimental de hemodiálise em cães (Canis familiaris). Dissertação (Mestrado). Faculdade de Medicina, Universidade Estadual Paulista, Botucatu. https://doi.org/10.1590/S0100-736X2010001000009

Meneses, A. M. C.; Caramori, J. C. T.; Brant, J. R. A. C.; Melchert, A.; Moutinho, F. Q.; Mamprim, M. J.; \& Barretti, P. (2002). Acesso vascular para hemodiálise em cães utilizando cateter duplo lúmen. In: Congresso Brasileiro De Clínicos Veterinários De Pequenos Animais. Anais... Brasília. http://dx.doi.org/10.1590/S0103-84782008000400016

Nakajima, Y., Hoshi, F., Higuchi, S., et al. The complete amino acid sequence of dog $\beta_{2}$ microglobulin. J. Vet. Med. Sci. 65 (5), 517-521. 10.1292/jvms.61.517

Nakajima, Y., Hoshi, F., Higuchi, S., et al. (2000). Determination of canine $\beta_{2}$ microglobulin in plasma and urine by Enzyme-linked Immunosorbent Assay. J. Vet. Med. Sci. 63(3), 343-345. 10.1292/jvms.63.343

Norman, G. R.; \& Streiner, D. L. (1994) Bioestatistics - The bare essentials. Mosby - year book, St. Louis.

Riella M. C. (1996) Princípios de Nefrologia e Distúrbios Hidroeletrolíticos. Guanabara Koogan.

Simmons, E. M., Himmelfarb, J., Sezer, M. T., et al. (2004). Plasma cytokine levels predict mortality in patients with acute renal failure. Kid. Int. 65, 13571365. https://doi.org/10.1111/j.1523-1755.2004.00512.x

Singh, N. P., Bansal, R., Thakur, A., et al. (2003). Effect of membrane composition on cytokine production and clinical symptoms during hemodialysis: a crossover study. Ren. Fail. 25(3), 419-430. https://doi.org/10.1081/JDI-120021154 